\title{
TREATING A CHILD WITH COUGH OR DIFFICULTY IN BREATHING AT DISTRICT HOSPIT ALS
}

\author{
Shrestha S $\mathbf{S}^{\star}$ Bhattarai $\mathrm{P}^{\star}$, Sharma M
}

\section{ABSTRACT}

Acute respiratory infections (ARI) are the commonest infections in children under the age of five years in Nepal. An under five child will have 4-6 episodes of ARI per year in Nepal. ARI episode does not differ much between developed and developing countries. Pneumonia is the commonest single cause that causes death in a child with respiratory infections in developing countries. Treating pneumonia is still confusing. This article re-visits the basic concept in the treatment of pneumonia.

\section{Key Words: Pneumonia, persistent, pneumonia, antibiotics, treatment.}

\section{INTRODUCTION}

It is the commonest cause of admission in under five children at Kanti Children's Hospital; approximately $70 \%$ of medical admissions are contributed by pneumonia. ${ }^{1}$ It is also one of the commonest causes of morbidity and mortality in Nepal and developing countries. ${ }^{2}$ Pneumonia is the leading cause of death in a child with acute respiratory infections. ${ }^{3}$

Pneumonia is diagnosed in a child with the history of cough or difficulty breathing having any one of the following signs: fast breathing (fast breathing cutoff rates per minute are as follows: young infant 60 or more; 2 months up to 12 months: 50 or more and up to 5 years: 40 or more) chest indrawing (observe for the lower chest indrawing during inspiration) ${ }^{4}$ and crepitations on auscultation.

Differential diagnosis of a child presenting with cough or difficulty breathing ${ }^{5}$ :

\section{Diagnosis}

Pneumonia
In favour

Fast breathing

Nasal flaring

Grunting

Headnodding
Lower chest wall in drawing

Bronchial breathing

Crepitations on auscultation

Cardiac failure

Raised jugular venous pressure Central cyanosis

Edema feet

Enlarged liver

Heart murmur

Gallop rhythm

Pneumothorax

Sudden onset

Hyper resonance chest on one side of chest.

Diminished air entry on the side of lesion

Shift of mediastinum (trachea, apex beat)

Pleural effusion, empyema Diminished movement of chest on affected side Intercostals fullness Stony dull on percussion Diminished breath sound on the side of lesion.

* Institute of Medicine, Maharajgunj, Kathmandu, Nepal.

Address for correspondence : Dr. Shiva Shrestha 


\begin{tabular}{|ll}
\hline Pericardial effusion & Edema feet \\
& Raised jugular venous pressure \\
& Apex beat not visible/not palpable \\
& Pulsus paradoxus \\
& Enlarge liver
\end{tabular}

Penumocystis pneumonia 2-6 month old child with central cyanosis

Hyper expanded chest

Fast breathing

Enlarged spleen, liver and lymph nodes

Wasting

\section{Bronchial Asthma}

H/O Recurrent cough/ wheezing

Noisy breathing

$\mathrm{H} / \mathrm{O}$ repeated hospital visit admission

Positive family history

$\mathrm{H} / \mathrm{O}$ atopy/eczema

Seasonal variation

Emotional child

\section{Broncheolitis}

\section{H/o viral URTI}

Symptoms more than signs

May go into CCF early

Age group 1mo-2 years

Respiratory distress

Hepatomegaly due to

hyperinflation

Treating pneumonia at Hospital is based on the following information:
Age of the patient.
Severity of signs
Mode of presentation
Duration of the illness
Associated diseases.
Availability of drugs.
Follow-up

\section{Age of the patient}

* Admit all patients below the age of 2 months (young infant).

* Assess if the young infant is able to suck well or not.

* If the young infant is taking half of the usual amount then insert a nasogastric tube (size 5 or 6) and give EBM $130 \mathrm{ml} / \mathrm{kg} / 24$ hours divided two to three hourly.

* If the young infant has severe chest indrawing/ cyanosis/ grunting/nasal flaring/lethargic then:
* Insert nasal canula and give oxygen at the rate of 2L/ $\min$.

(Measure the distance from the tragus of ear to the tip of the nose and make it half. Mark this length on the canula from the distal end. Insert through the nostril up to the mark and fix it on the forehead with tape.)

Keep the young infant warm and in slightly propped up positions. (educate the mother.)

* Give Gentamicin IM (6mg/kg single dose) plus Procaine penicillin IM (25-50,000 units/kg single dose) daily for one week ${ }^{6}$.

DO NOT USE IV FLUID UNLESS THERE IS DEHYDRATION (inappropriate ADH secretion is one of the important complication which may cause cardiac failure)

\section{Severity of signs}

Admit all patients with any of the following severe signs: Severe chest indrawing, flaring alae nasi, grunting, stridor, audible wheeze, cyanosis, lethargic or unconscious (size of the consolidation in the chest $\mathrm{x}$-ray is not the criteria for the admission)

Give oxygen as mentioned earlier. Using oxygen will reduce the mortality by $70 \%$

Give IV fluid $1 / 5^{\text {th }}$ Normal saline in $10 \%$ dextrose (half of the total fluid requirement if the child is not able to drink) for 24 hours.

Give IV ceftriaxone $50 \mathrm{mg} / \mathrm{kg}$ twice daily (if cannot afford give IV chloramphenicol $50 \mathrm{mg} / \mathrm{kg} /$ day divided six hourly plus Penicillin G potassium or sodium 100,000 units/kg in divided every six hourly) ${ }^{7}$. It is important to realize that the chloramphenicol if given orally will have the same MIC in the blood as compared to the IV. Chloramphenicol should never be given IM.

Assess if the patient is able to drink. If the patient is able to drink and does not have the above-mentioned signs except fast breathing then use oral antibiotics as mentioned below:

If they can afford use amoxycillin plus clavulanic acid ( $15 \mathrm{mg} / \mathrm{kg} / /$ dose of amoxycillin 8 hourly) for 5 days or give oral amoxycillin (20mg/kg/dose eight hourly) for five days and send home with counseling for fever, food and when to return.

If the child has wheeze: give nebulized salbutamol as follows:

* Place the $2.5 \mathrm{mg}$ of salbutamol solution $(0.5 \mathrm{ml}$ of the $5 \mathrm{mg} / \mathrm{ml}$ ) and $2-4 \mathrm{ml}$ of sterile saline in the nebulizer compartment and treat the child until the liquid is almost all used up. 
* Auscultate the child after the nebulizer and if the wheeze has improved teach parent about the use of salbutamol by metered dose inhaler with spacer device.

* Once the child has improved to be discharged, oral salbutamol can be given if inhaled salbutamol is not available. ${ }^{8}$

The dose is: Age 2-12 months: $1 \mathrm{mg} 6-8$ hourly. Age 12 mo - 5 years: $2 \mathrm{mg} 6-8$ hourly (strengthavailable: syrp: $2 \mathrm{mg} / 5 \mathrm{ml}$; tablet: $2,4 \mathrm{mg} / \mathrm{tab}$ )

* If the child has wheeze which is severe and more than 24 hours use oral prednisolone $1 \mathrm{mg} / \mathrm{kg}$ once a day for 3 days.

* If the child does not improve after three doses of nebulized salbutamol use aminophylline in the following doses:

\section{Bolus dose:}

$5-6 \mathrm{mg} / \mathrm{kg}$ (up to maximum of $300 \mathrm{mg}$ ) followed by a maintenance dose of $5 \mathrm{mg} / \mathrm{kg}$ every 6 hours. This IV dose has to be given diluted 4 times with 5\% dextrose over 30 minutes.

\section{Mode of presentation}

Pneumonia in older children often present in many ways:

A. Typical presentations (predominantly respiratory signs): fast breathing, chest indrawing, and cyanosis.

B. Atypical presentations (single or in combinations):
i. Acute abdominal pain
ii. Acute pain in chest or shoulder
iii. Convulsion
iv. Fever
v. Meningism

\section{Duration of illness}

If the duration of the illness is more than one week and the child does not have severe signs assess for reactive airway disease, tuberculosis, bronchiectasis, eosinophilia etc. Investigate with CBC, ESR, chest-x-ray and mantoux test. Counsel the parents: cause of cough, necessity of investigation and follow-up. It is important to differentiate recurrent and persistent pneumonia. Presence of a symptom free interval during which chest radiographs show clearing of infiltrates, suggests recurrent infection. At times persistent infections may present as recurrent infections because of inadequate or appropriate therapy. Congenital malformations, aspirations, defect in the clearance of airways secretions and disorders of local/ systemic immunity are important causes of recurrent or persistent infections.

\section{Associated diseases}

Pneumonia often associates with wheeze. If the wheeze is present a rapid acting bronchodilator should be used along with the oxygen.

Admit the patient with pneumonia if the following associated conditions are present: severe malnutrition, congenital cyanotic heart disease, sepsis, seizure in the present episode, prupuric rashes, some or severe signs of dehydration ${ }^{9}$. These cases needs further workup and specific treatment along with the treatment for pneumonia.

\section{Availability of drugs}

For OPD treatment, if affordable the use of amoxycillin plus clavulanic acid $(15 \mathrm{mg} / \mathrm{kg} / /$ dose of amoxycillin 8 hourly for 5 days) is recommended. If not affordable oral cotrimoxazole (10mg trimethoprim plus $50 \mathrm{mg}$ sulfamethoxazole $/ \mathrm{kg} / 24$ hour divided every 12 hourly) or amoxycillin $(20 \mathrm{mg} / \mathrm{kg} /$ dose every 8 hourly) is also equally effective.

For inpatient: if patient can afford, use of parental ceftriaxone in all age group is recommended. If it is not affordable use of crystalline penicillin plus gentamicin for young infant and chloramphenicol plus penicillin in older children with severe pneumonia is recommended as mentioned earlier.

\section{Follow-up}

Observe daily: respiratory rate, chest indrawing, feeding, convulsion, rash.

If the condition is worse after 24 hours following workup is needed:

CBC, chest $\mathrm{x}$ - ray. If there is convulsion do LP

These results must be obtained immediately. Observe for shift to the left, and in blood picture leukaemoid reaction, pneumothorax, and meningitis.

On the third day: If the condition is the same following work up is needed: CBC, ESR, Mantoux test, Chest xray, history of contact or foreign body aspiration, change the antibiotics to gentamycin and cloxacillin. Observe till the results are available. Follow-up should be done daily as mentioned earlier. One of the common causes of persisting fast breathing in a child with pneumonia after 48 hours of treatment is congestive cardiac failure. Therefore if the child has signs suggestive of cardiac failure use diuretics (furosamide $1 \mathrm{mg} / \mathrm{kg} / \mathrm{day}$ and oral lanoxin). In this case the symptoms should improve after 24 hours of this therapy. 
If the condition is better switch over to oral antibiotics except for young infants. Continue parental antibiotics in young infants for one week if there are no complications.

Older children can be discharged home the next day of oral antibiotics. The total duration of antibiotics (including oral and parenteral) should be 10 days. Children with pneumonia should not be prescribed cough mixtures containing cough suppressants and antihistamines.

Once the patient is discharged from the hospital it is essential to educate the parents and be sure that the parent understands the following points:

1. How to give the drug?

2. When to give the drug?

3. When to return?

\section{Cost of treatment}

It is very important to consider the cost of the treatment because many drugs are costly and the result will be same. Below is the example of the cost of the treatment for a child of $10 \mathrm{~kg}$ from OPD. ${ }^{9}$

\section{Name of the brand and preparation}

Approximate cost of the drug for 5-day treatment

Amoxicillin with clavulanate Pot.

Rs. 436/-

Cotrimoxazole (Syrup)

Rs. $51 /-$

Cotrimoxazole( Paed. Tablet)

Rs. $25 / 50$

\section{CONCLUSION}

Pneumonia is the one of the common respiratory diseases that kills approximately 40,000 children annually in Nepal. Pneumonia should be treated effectively and adequately in the health care facility to prevent complications or mortality. Even in the presence of complications e.g. CCF, SIADH, and fluid overload patient can be treated provided physicians are aware of the nature of the complications and its presentations. Administration of IV fluids should be discouraged unless patient is dehydrated or lethargic. If available, oxygen inhalation would be very helpful in patients with increasing respiratory effort and cyanosis.
Respiratory rate is the key sign to diagnose pneumonia in the peripheral hospitals. Increasing respiratory difficulty as evidenced by high respiratory rate, nasal flaring, lower chest wall in drawing, cyanosis, difficulty in feeding, sweating over the forehead, and diminished urinary output are few of the common clinical signs to identify severe pneumonia. Recognition of these features in time will lead to a physician to reach in the diagnosis and proper management of the case.

\section{REFERENCES}

1. Acute respiratory infections in Nepal: Hospital based study. J.Nep. Paed. Society. 5:1: 1986.

2. Pandey $M R$, Sharma PR, Gubhaju BB et al. Impact of a pilot acute respiratory infection (ARI) control programme in a rural community of the hill region of Nepal. Annals of Tropical Pediatrics. 1989: 9:212-220.

3. Selwyn BJ on behalf of the Coordinated Data Group of the BOSTID Researchers. The epidemiology of acute respiratory infections in young children: Comparison of findings from several developing countries. Rev. Infect Dis 1990; 12 (Suppl 8) :S870-S888.

4. Acute respiratory infections in children case management in small hospitals in developing countries. A manual for doctors and other senior health workers. Programme for the control of acute respiratory infections. WHO/ARI/90.5; WHO Geneva.

5. Rubin BK. The evaluation of a child with recurrent chest infections. Pediatr Infect Dis J, 1985; 4:88-98

6. Antibiotics in the treatment of acute respiratory infections in young children. Programme for the control of the acute respiratory infections. WHO/ARI/90.10 WHO Geneva.

7. Bronchodilators and other medications for the treatment of wheeze-associated illnesses in young children. Programme for the control of acute respiratory infections. WHO/ARI/ 93.29. WHO Geneva.

8. Management of the child with a serious infection or severe malnutrition. Gidelines for care at the first referral level in developing countries. WHO. Department of Child Health. Geneva. 2000; 19-37.

9. Current Index of Medical Specialties (CIMS) April 2004. 